\title{
RIGIDITY RESULTS, INVERSE CURVATURE FLOWS AND ALEXANDROV-FENCHEL TYPE INEQUALITIES IN THE SPHERE*
}

\author{
MATTHIAS MAKOWSKI AND JULIAN SCHEUER ${ }^{\dagger}$
}

\begin{abstract}
We prove a rigidity result in the sphere which allows us to generalize a result about smooth convex hypersurfaces in the sphere by Do Carmo and Warner to convex $C^{2}$-hypersurfaces. We apply these results to prove $C^{1, \beta}$-convergence of inverse $F$-curvature flows in the sphere to an equator in $\mathbb{S}^{n+1}$ for embedded, closed and strictly convex initial hypersurfaces. The result holds for large classes of curvature functions including the mean curvature and arbitrary powers of the Gauss curvature. We use this result to prove some Alexandrov-Fenchel type inequalities.
\end{abstract}

Key words. Rigidity, inverse curvature flows, sphere, Alexandrov-Fenchel-inequality.

AMS subject classifications. 35J60, 53C21, 53C24, 53C44, 58J05.

1. Introduction. This work deals with geometric problems in the $(n+1)$ dimensional unit sphere $\mathbb{S}^{n+1} \subset\left\{x \in \mathbb{R}^{n+2}:|x|=1\right\}$. We assume $n \geq 2$, unless stated otherwise. We are interested in the connection between (analytically) convex hypersurfaces and (geodesically) convex bodies. The notion of convexity of sets is significantly more subtle than in Euclidean space, due to the existence of focal points in the sphere.

A very well known result in this direction by do Carmo and Warner, [5, Theorem 1.1], is the following.

Theorem 1 (Do Carmo, Warner, [5]). Let $x: M^{n} \rightarrow \mathbb{S}^{n+1}$ be an isometric immersion of a compact, connected, orientable $n$-dimensional $C^{\infty}$-Riemannian manifold into the $(n+1)$-sphere of sectional curvature equal to one and assume that all sectional curvatures of $M^{n}$ are greater than or equal to one. Then $x$ is an embedding, $M^{n}$ is diffeomorphic to $\mathbb{S}^{n}$ and $x\left(M^{n}\right)$ is either totally geodesic or contained in an open hemisphere. In the latter case $x\left(M^{n}\right)$ is the boundary of a convex body in $\mathbb{S}^{n+1}$.

Also compare [1], which deals with strictly convex hypersurfaces. We will show that some parts of this result can be generalized to non-smooth, geodesically convex bodies in the sphere. In particular we will prove the following result, for the exact definitions of weakly convex bodies see Section 3 .

THEOREM 1.1. Let $n \geq 1$ and $\hat{M} \subset \mathbb{S}^{n+1}$ be a weakly convex body in a hemisphere. Let $x_{0} \in \mathbb{S}^{n+1}$ be such that $\hat{M}$ is contained in the closed hemisphere $\mathcal{H}\left(x_{0}\right)$ with equator $\mathcal{S}\left(x_{0}\right)$. Suppose that $\hat{M}$ satisfies an interior sphere condition at all points $p \in \hat{M} \cap \mathcal{S}\left(x_{0}\right)$. Then either $\hat{M}$ is equal to $\mathcal{H}\left(x_{0}\right)$ or $\hat{M}$ is contained in an open hemisphere.

With the help of this result, we prove that the strong regularity assumption in [5] is not necessary.

Corollary 1.2. Let $M \subset \mathbb{S}^{n+1}$ be an embedded, closed, connected and convex $C^{2}$-hypersurface. Then $M$ is either an equator or $M$ is contained in an open hemisphere and bounds a convex body.

${ }^{*}$ Received July 21, 2014; accepted for publication April 28, 2015.

$\dagger$ Corresponding author. Albert-Ludwigs-Universität, Mathematisches Institut, Eckerstr. 1, 79104 Freiburg, Germany (julian.scheuer@math.uni-freiburg.de). During preparation of this work the second author had been supported by the "Deutsche Forschungsgemeinschaft" (DFG). 
We apply these rigidity results to treat an inverse curvature flow in the sphere $\mathbb{S}^{n+1}$ of the form

$$
\begin{aligned}
\dot{x} & =-\Phi(F) \nu, \\
x(0) & =x_{0},
\end{aligned}
$$

where $x_{0}: \mathbb{S}^{n} \rightarrow \mathbb{S}^{n+1}$ is the embedding of an initial hypersurface $M_{0}:=x_{0}\left(\mathbb{S}^{n}\right)$ of class $C^{4, \alpha}$ for some $0<\alpha<1$, which is furthermore required to be strictly convex. $\nu$ is the corresponding outer normal, $\Phi \in C^{\infty}\left(\mathbb{R}_{+}, \mathbb{R}\right), \Phi(x)=-x^{-p}, p>0, F$ is a curvature function evaluated at the principal curvatures of the flow hypersurfaces $M_{t}$ and $x(t)$ denotes the embedding of $M_{t}$. We will show that under certain assumptions, cf. 1.3, the flow exists up to a finite maximal time and converges in $C^{1, \beta}$ to the embedding of an equator.

Curvature flows and their application to geometric inequalities have been treated for over thirty years. Following the ground breaking work by Huisken, [16], who considered the mean curvature flow, also inverse, or expanding flows have been considered. Here the works on inverse curvature flows by Gerhardt, [9], as well as Urbas, [27], have to be mentioned, where also non-convex hypersurfaces were considered. Similar results have been shown in other ambient spaces and for general $p$-homogeneous curvature functions, e.g. [12], [13], or [25].

We consider a large class of curvature functions. We allow other powers $p$ than 1 , in particular our result holds for arbitrary powers of the Gaussian curvature without further pinching assumptions. The detailed assumptions on the curvature function are listed below, whereafter we state the convergence result.

Assumption 1.3. Suppose $F \in C^{2, \alpha}\left(\Gamma_{+}\right), 0<\alpha<1$, is a symmetric function, where $\Gamma_{+}=\left\{\kappa=\left(\kappa_{i}\right) \in \mathbb{R}^{n}: \kappa_{i}>0 \forall i \in\{1, \ldots, n\}\right\}$. We need the following assumptions on the curvature function $F$ :

- $F$ is positively homogeneous of degree 1 , i.e. $\forall \kappa \in \Gamma_{+}, \forall \lambda \in \mathbb{R}_{+}: F(\lambda \kappa)=$ $\lambda F(\kappa)$.

- $F$ is strictly increasing in each argument: $\forall i \in\{1, \ldots, n\}, \forall \kappa \in \Gamma_{+}$there holds $F_{i}(\kappa)=\frac{\partial F}{\partial \kappa_{i}}(\kappa)>0$.

- $F$ is positive, $F_{\mid \Gamma_{+}}>0$, and $F$ is normalized, $F(1, \ldots, 1)=n$.

- Either:

(i) $F$ is concave and inverse concave, i.e. $F_{-1}\left(\kappa_{i}\right):=\frac{1}{F\left(\kappa_{i}^{-1}\right)}$ is concave.

(ii) $F$ is concave and $F$ approaches zero on the boundary of $\Gamma_{+}$.

- If $p \neq 1$, we assume (ii) is valid.

The most important examples of curvature functions $F$ being concave and inverse concave are $\left(\frac{H_{k}}{H_{l}}\right)^{\frac{1}{k-l}}, n \geq k>l \geq 0$, or the power means $\left(\sum_{i=1}^{n} \kappa_{i}^{r}\right)^{\frac{1}{r}}$ for $|r| \leq 1$. For a proof of the inverse concavity of these functions see the proofs of $[2$, Theorem 2.6, Theorem 2.7].

Our exact result concerning the curvature flows is:

Theorem 1.4. Let $0<\alpha<1$. Let $\mathbb{S}^{n} \hookrightarrow M_{0} \subset \mathbb{S}^{n+1}$ be an embedded, closed, connected and strictly convex hypersurface of class $C^{4, \alpha}$. Let $F$ be a curvature function satisfying Assumption 1.3. Then there exists a finite time $0<T^{*}<\infty$ and a unique curvature flow

$$
x \in H^{2+\alpha, \frac{2+\alpha}{2}}\left(\left[0, T^{*}\right) \times \mathbb{S}^{n}, \mathbb{S}^{n+1}\right),
$$


which satisfies the flow equation

$$
\begin{aligned}
\dot{x} & =F^{-p} \nu \\
x(0) & =M_{0},
\end{aligned}
$$

where $0<p<\infty, \nu(t, \xi)$ is the outward normal to $M_{t}=x(t, M)$ at $x(t, \xi)$ and there exists $0<t_{0}<T^{*}$ such that the leaves $M_{t}, t_{0} \leq t<T^{*}$, are graphs over some suitable equator $\mathcal{S}\left(x_{0}\right), x_{0} \in \mathbb{S}^{n+1}$,

$$
M_{t}=\operatorname{graph} u(t, \cdot),
$$

where $u$ is the radial distance to $x_{0}$. For $t \rightarrow T^{*}$, the functions $u(t, \cdot)$ converge to $\frac{\pi}{2}$ in $C^{1, \beta}\left(\mathbb{S}^{n}\right)$ for arbitrary $0<\beta<1$ and we have for $1 \leq q<\infty$, that

$$
\int_{M_{t}} H^{q} \rightarrow 0, t \rightarrow T^{*}
$$

In this theorem, $H^{2+\alpha, \frac{2+\alpha}{2}}\left(\left[0, T^{*}\right) \times \mathbb{S}^{n}, \mathbb{S}^{n+1}\right)$ denotes the parabolic Hoelder space as in [10, Definition 2.5.2].

Recently, Gerhardt also considered inverse curvature flows of strictly convex hypersurfaces in $\mathbb{S}^{n+1}$ by curvature functions satisfying the assumptions of 1.3(i) in case $p=1$, see [14]. He obtains smooth convergence of the flow to an equator. However, his methods substantially differ from ours.

Theorem 1.4 allows us to prove Alexandrov-Fenchel type inequalities in the sphere, namely:

TheOREM 1.5. Let $M \subset \mathbb{S}^{n+1}$ be an embedded, closed, connected and convex $C^{2}$-hypersurface of the sphere. Then we have the inequality

$$
\left(\tilde{V}_{1}(M)\right)^{2} \geq\left(\tilde{V}_{0}(M)\right)^{2\left(\frac{n-1}{n}\right)}-\left(\tilde{V}_{0}(M)\right)^{2},
$$

and equality holds if and only if $M$ is a geodesic sphere.

Furthermore, if $n \geq 3$, we have the inequality

$$
\tilde{V}_{2}(M) \geq\left(\tilde{V}_{0}(M)\right)^{\frac{n-2}{n}}-\tilde{V}_{0}(M)
$$

and equality holds if and only if $M$ is a geodesic sphere.

Let $k \in \mathbb{N}_{+}$with $2 k+1 \leq n$ and let $\hat{M}$ be the convex body enclosed by $M$. Then we have the inequality

$$
W_{2 k+1}(\hat{M}) \geq \frac{\omega_{n}}{n+1} \sum_{i=0}^{k}(-1)^{i} \frac{n-2 k}{n-2 k+2 i}\left(\begin{array}{c}
k \\
i
\end{array}\right)\left(\frac{n+1}{\omega_{n}} W_{1}(\hat{M})\right)^{\frac{n-2 k+2 i}{n}}
$$

and equality holds if and only if $M$ is a geodesic sphere.

Here $\tilde{V}_{k}(M)$ denotes, up to a constant, the $k$-th mean curvature integral and is defined by

$$
\tilde{V}_{k}(M):=\omega_{n}^{-1} \int_{M} \tilde{H}_{k} d \mu,
$$


where $\tilde{H}_{k}:=\frac{H_{k}}{\left(\begin{array}{c}n \\ k\end{array}\right)}$ are the normalized elementary symmetric polynomials and $\omega_{n}:=$ $\left|\mathbb{S}^{n}\right| . W_{k}(\hat{M})$ denotes the $k$-th quermassintegral of $\hat{M}$, see Section 7 for a definition. For a more detailed account of the mean curvature integrals and their relation to the quermassintegrals in spaces of constant curvature, see for example [26]. Especially inequality (1.7) shows that the geometric inequalities for the quermassintegrals resemble the corresponding inequalities in hyperbolic space, see [8, Theorem 1.3]. After submission of this paper, in [29] Wei and Xiong obtained similar geometric inequalities in space forms, where in parts of their paper they also use flow methods.

Curvature flows have shown to be a useful method to obtain geometric inequalities. Probably the most known result in this direction is the proof of the Riemannian Penrose inequality by Huisken and Ilmanen in [17] using an inverse mean curvature flow in asymptotically flat 3 -manifolds.

Also Alexandrov-Fenchel type inequalities have been proven using curvature flows: In Euclidean space, McCoy showed in [22], that the Alexandrov-Fenchel inequalities for strictly convex hypersurfaces can be deduced from a mixed-volume preserving curvature flow. In 2009, Guan and Li, [15], used inverse F-curvature flows in Euclidean space to show these inequalities for $k$-convex, starshaped domains. Recently, the first author transferred the results about mixed-volume preserving curvature flows in Euclidean space from [22] to the hyperbolic space in [21]. Wang and Xia used these results in [28] to obtain the Alexandrov-Fenchel inequalities for horospherically convex hypersurfaces in hyperbolic space. Some of these inequalities have also been shown in hyperbolic space by using inverse $F$-curvature flows, see for example [7], [8], [4].

2. Setting and general facts. Now we state some general facts about hypersurfaces, especially those that can be written as graphs. We basically follow the description of [12] and [25], but restrict to Riemannian manifolds. For a detailed discussion we refer to [10].

Let $N=N^{n+1}$ be Riemannian and $M=M^{n} \hookrightarrow N$ be a hypersurface. The geometric quantities of $N$ will be denoted by $\left(\bar{g}_{\alpha \beta}\right),\left(\bar{R}_{\alpha \beta \gamma \delta}\right)$ etc., where greek indices range from 0 to $n$. Coordinate systems in $N$ will be denoted by $\left(x^{\alpha}\right)$. Quantities for $M$ will be denoted by $\left(g_{i j}\right),\left(h_{i j}\right)$ etc., where latin indices range from 1 to $n$ and coordinate systems will generally be denoted by $\left(\xi^{i}\right)$, unless stated otherwise.

Covariant differentiation will usually be denoted by indices, e.g. $u_{i j}$ for a function $u: M \rightarrow \mathbb{R}$, or, if ambiguities are possible, by a semicolon, e.g. $h_{i j ; k}$. Usual partial derivatives will be denoted by a comma, e.g. $u_{i, j}$.

Let $x: M \hookrightarrow N$ be an embedding and $\left(h_{i j}\right)$ be the second fundamental form with respect to a differentiable normal $-\nu$, i.e. we have the Gaussian formula

$$
x_{i j}^{\alpha}=-h_{i j} \nu^{\alpha}
$$

where $\nu$ is a differentiable normal, the Weingarten equation

$$
\nu_{i}^{\alpha}=h_{i}^{k} x_{k}^{\alpha}
$$

the Codazzi equation

$$
h_{i j ; k}-h_{i k ; j}=\bar{R}_{\alpha \beta \gamma \delta} \nu^{\alpha} x_{i}^{\beta} x_{j}^{\gamma} x_{k}^{\delta}
$$

and the Gauß equation

$$
R_{i j k l}=\left(h_{i k} h_{j l}-h_{i l} h_{j k}\right)+\bar{R}_{\alpha \beta \gamma \delta} x_{i}^{\alpha} x_{j}^{\beta} x_{k}^{\gamma} x_{l}^{\delta} .
$$


Now assume that $N=(a, b) \times S_{0}$, where $S_{0}$ is compact Riemannian and that there is a Gaussian coordinate system $\left(x^{\alpha}\right)$ such that

$$
d \bar{s}^{2}=e^{2 \psi}\left(\left(d x^{0}\right)^{2}+\sigma_{i j}\left(x^{0}, x\right) d x^{i} d x^{j}\right),
$$

where $\sigma_{i j}$ is a Riemannian metric, $x=\left(x^{i}\right)$ are local coordinates for $\mathcal{S}_{0}$ and $\psi: N \rightarrow \mathbb{R}$ is a function.

Let $M=\operatorname{graph} u_{\mid \mathcal{S}_{0}}$ be a hypersurface

$$
M=\left\{\left(x^{0}, x\right): x^{0}=u(x), x \in \mathcal{S}_{0}\right\},
$$

then the induced metric has the form

$$
g_{i j}=e^{2 \psi}\left(u_{i} u_{j}+\sigma_{i j}\right)
$$

with inverse

$$
g^{i j}=e^{-2 \psi}\left(\sigma^{i j}-v^{-2} u^{i} u^{j}\right),
$$

where $\left(\sigma^{i j}\right)=\left(\sigma_{i j}\right)^{-1}, u^{i}=\sigma^{i j} u_{j}$ and

$$
v^{2}=1+\sigma^{i j} u_{i} u_{j} \equiv 1+|D u|^{2} .
$$

We use, especially in the Gaussian formula, the normal

$$
\left(\nu^{\alpha}\right)=v^{-1} e^{-\psi}\left(1,-u^{i}\right) .
$$

Looking at $\alpha=0$ in the Gaussian formula, we obtain

$$
e^{-\psi} v^{-1} h_{i j}=-u_{i j}-\bar{\Gamma}_{00}^{0} u_{i} u_{j}-\bar{\Gamma}_{0 i}^{0} u_{j}-\bar{\Gamma}_{0 j}^{0} u_{i}-\bar{\Gamma}_{i j}^{0}
$$

and

$$
e^{-\psi} \bar{h}_{i j}=-\bar{\Gamma}_{i j}^{0}
$$

where covariant derivatives are taken with respect to $g_{i j}$.

In our special situation $N=\mathbb{S}^{n+1}$ let $x_{0} \in \mathbb{S}^{n+1}$, then by introducing geodesic polar coordinates we derive a representation of the metric in the form

$$
d \bar{s}^{2}=d r^{2}+\sin ^{2} r \sigma_{i j} d x^{i} d x^{j}
$$

where $\sigma_{i j}$ is the canonical metric of $\mathbb{S}^{n}$ and $0<r<\pi$. Then we obtain for a geodesic sphere given by a constant graph $u \equiv r$ with $0<r<\pi$ that $\bar{h}_{i j}=\frac{\bar{H}}{n} \bar{g}_{i j}$ and

$$
\frac{\bar{H}}{n}(r)=\frac{\cos r}{\sin r} \text {. }
$$

Using [5, Theorem 1.1], we conclude for an embedding of a smooth, strictly convex, closed hypersurface $M$, that it is contained in an open hemisphere and thus it can be written as a graph over $\mathbb{S}^{n}$ in the previously described coordinate system, i.e.

$$
M=\operatorname{graph} u_{\mid \mathcal{S}_{0}} .
$$

Now we want to give some elementary facts about the curvature functions. Firstly we provide the definition of these functions and mention some identifications, which will be used in the sequel without explicitly stating them again. 
Definition 2.1. Let $\Gamma \subset \mathbb{R}^{n}$ be an open, convex, symmetric cone, i.e.

$$
\left(\kappa_{i}\right) \in \Gamma \Longrightarrow\left(\kappa_{\pi i}\right) \in \Gamma \quad \forall \pi \in \mathcal{P}_{n}
$$

where $\mathcal{P}_{n}$ is the set of all permutations of order $n$. Let $f \in C^{m, \alpha}(\Gamma), m \in \mathbb{N}, 0 \leq \alpha \leq 1$, be symmetric, i.e.,

$$
f\left(\kappa_{i}\right)=f\left(\kappa_{\pi i}\right) \quad \forall \pi \in \mathcal{P}_{n}
$$

Then $f$ is said to be a curvature function of class $C^{m, \alpha}$. For simplicity we will also refer to the pair $(f, \Gamma)$ as a curvature function.

Now denote by $\mathbf{S}$ the symmetric endomorphisms of $\mathbb{R}^{n}$ and by $\mathbf{S}_{\Gamma}$ the symmetric endomorphisms with eigenvalues belonging to $\Gamma$, an open subset of $\mathbf{S}$. If $(f, \Gamma)$ is a smooth curvature function, we can define a mapping

$$
\begin{aligned}
F: \mathbf{S}_{\Gamma} & \rightarrow \mathbb{R}, \\
A & \mapsto f\left(\kappa_{i}\right),
\end{aligned}
$$

where the $\kappa_{i}$ denote the eigenvalues of $A$. For the relation between these different notions, especially the differentiability properties and the relation between their derivatives, see [10, Chapter 2.1]. Since the differentiability properties are the same for $f$ as for $F$ in our setting, see [10, Theorem 2.1.20], we do not distinguish between these notions and always write $F$ for the curvature function. Hence at a point $x$ of a hypersurface we can consider a curvature function $F$ as a function defined on a cone $\Gamma \subset \mathbb{R}^{n}, F=F\left(\kappa_{i}\right)$ for $\left(\kappa_{i}\right) \in \Gamma$ (representing the principal curvatures at the point $x$ of the hypersurface), as a function depending on $\left(h_{i}^{j}\right), F=F\left(h_{i}^{j}\right)$, or as a function depending on $\left(h_{i j}\right)$ and $\left(g_{i j}\right), F=F\left(h_{i j}, g_{i j}\right)$. However, we distinguish between the derivatives with respect to $\Gamma$ or $\mathbf{S}$. We briefly summarize our notation and important properties:

For a smooth curvature function $F$ we denote by $F^{i j}=\frac{\partial F}{\partial h_{i j}}$, a contravariant tensor of order 2 , and $F_{i}^{j}=\frac{\partial F}{\partial h_{j}^{i}}$, a mixed tensor, contravariant with respect to the index $j$ and covariant with respect to $i$. We also distinguish the partial derivative $F_{, i}=\frac{\partial F}{\partial \kappa_{i}}$ and the covariant derivative $F_{; i}=F^{k l} h_{k l ; i}$. Furthermore $F^{i j}$ is diagonal if $h_{i j}$ is diagonal and in such a coordinate system there holds $F^{i i}=\frac{\partial F}{\partial \kappa_{i}}$. For a relation between the second derivatives see [10, Lemma 2.1.14]. Finally, if $F \in C^{2}(\Gamma)$ is concave (convex), then $F$ is also concave (convex) as a curvature function depending on $\left(h_{i j}\right)$.

3. Rigidity results. In this section we want to prove the rigidity result, Theorem 1.1 and Corollary 1.2. First we need some definitions, which also apply to the case $n \geq 1$.

\section{Definition 3.1.}

(i) For a point $x \in \mathbb{S}^{n+1}$ we will denote the closed hemisphere with center in $x$ by $\mathcal{H}(x)$

$$
\mathcal{H}(x):=\left\{p \in \mathbb{S}^{n+1}:\langle p, x\rangle \geq 0\right\}
$$

where $\langle\cdot, \cdot\rangle$ is the scalar product in $\mathbb{R}^{n+2}$, and the corresponding equator by $\mathcal{S}(x):=\mathcal{H}(x) \backslash \operatorname{int} \mathcal{H}(x)$. 
(ii) For points $p, q \in \mathbb{S}^{n+1}, \hat{\gamma}_{p, q}:[0, L] \rightarrow \mathbb{S}^{n+1}$ will denote a $C^{1}$ parametrization by arc length of the geodesic segment $\hat{\Gamma}_{p, q}$ with $\hat{\gamma}_{p, q}(0)=p$ and $\hat{\gamma}_{p, q}(L)=q$. The geodesic segment is not unique if $\operatorname{dist}(p, q)=\pi$.

(iii) For $x \in \mathbb{S}^{n+1}$ the stereographic projection, which maps $x$ to 0 will be denoted by $\mathcal{P}_{x}: \mathbb{S}^{n+1} \backslash\{-x\} \rightarrow \mathbb{R}^{n+1}$.

DEFINITION 3.2.

(i) Let $\hat{M} \subset \mathbb{S}^{n+1}$ be a set. We say that $\hat{M}$ is a weakly convex set in $\mathbb{S}^{n+1}$ [in a hemisphere], if [there exists $x \in \mathbb{S}^{n+1}$ such that $\hat{M} \subset \mathcal{H}(x)$ and] for arbitrary $p, q \in \hat{M}$ there exists a minimizing geodesic $\hat{\Gamma}_{p, q}$ connecting $p$ and $q$, which is contained in $\hat{M}$.

(ii) Let $\hat{M} \subset \mathbb{S}^{n+1}$ be a set. We say that $\hat{M}$ is a convex set in $\mathbb{S}^{n+1}$ [in a hemisphere], if [there exists $x \in \mathbb{S}^{n+1}$ such that $\hat{M} \subset \mathcal{H}(x)$ and] for arbitrary $p, q \in \hat{M}$ all minimizing geodesics $\hat{\Gamma}_{p, q}$ connecting $p$ and $q$ [and contained in $\mathcal{H}(x)$ ] are contained in $\hat{M}$.

(iii) We say that $\hat{M} \subset \mathbb{S}^{n+1}$ is a (weakly) convex body [in a hemisphere], if it is a compact, (weakly) convex set [in a hemisphere] with nonempty interior.

(iv) A set $M \subset \mathbb{S}^{n+1}$ is a closed, geodesically convex hypersurface, if there exists a convex body $\hat{M} \subset \mathbb{S}^{n+1}$ in a hemisphere, such that $M=\partial \hat{M}$. The set $\hat{M}$ is called the convex body of $M$.

(v) Let $x \in \mathbb{S}^{n+1}$. Let $\hat{M} \subset \mathbb{S}^{n+1}$ be a set with $\overline{\hat{M}} \subset \mathbb{S}^{n+1} \backslash\{-x\}$. We say that $\hat{M}$ satisfies an interior sphere condition at a point $p \in \overline{\hat{M}}$ with respect to $x$, if the set $\mathcal{P}_{x}(\bar{M}) \subset \mathbb{R}^{n+1}$ satisfies an interior sphere condition at $\mathcal{P}_{x}(p)$.

REMARK 3.3. The following observations have to be made:

(i) Let $M \subset \mathbb{S}^{n+1}$ be a closed, geodesically convex hypersurface. Then the convex body of $M$ is not unique, as can be seen by looking at $M=\mathcal{S}(x)$, where $x \in \mathbb{S}^{n+1}$ is arbitrary.

(ii) Note that there are different notions of convexity in the sphere: we do not demand that a geodesic connecting two points in the convex body has to be unique. It is well-known, see also Lemma 3.8 , that a convex body $\hat{M}$ in the sphere, which does not contain a pair of antipodal points is contained in an open hemisphere. If on the other hand the convex body $\hat{M}$ in the sphere contains antipodal points, then it follows from the definition that $\hat{M}=\mathbb{S}^{n+1}$.

REMARK 3.4. The following observations can be found in [10, p. 278, 279]. Let $x_{0} \in \mathbb{S}^{n+1}$. Defining

$$
\rho=2 \tan \frac{r}{2}
$$

where $r$ is the geodesic distance to $x_{0}$, we obtain a representation of the spherical metric as

$$
d \bar{s}^{2}=\frac{1}{\left(1+\frac{1}{4} \rho^{2}\right)^{2}}\left(d \rho^{2}+\rho^{2} \sigma_{i j} d \xi^{i} d \xi^{j}\right) \equiv e^{2 \psi} \hat{g}_{\alpha \beta},
$$

where $\hat{g}$ denotes the Euclidean metric in $\mathbb{R}^{n+1}$. A point $q \in \mathbb{S}^{n+1}$ is contained in $\mathcal{H}\left(x_{0}\right)$ if and only if $r \leq \frac{\pi}{2}$, which is equivalent to $\rho \leq 2$.

A $C^{2}$-hypersurface $M \subset \mathbb{S}^{n+1} \backslash\left\{-x_{0}\right\}$ can be seen as embedded in Euclidean space using the conformally flat parametrization of $\mathbb{S}^{n+1}$ via stereographic projection. 
We will denote the projected hypersurface by $\mathcal{M}$. The second fundamental form $h_{j}^{i}$ of $M$ and the corresponding Euclidean quantity $\hat{h}_{j}^{i}$ are related by

$$
e^{\psi} h_{j}^{i}=\hat{h}_{j}^{i}+\psi_{\alpha} \hat{\nu}^{\alpha} \delta_{j}^{i}
$$

where $\hat{\nu}$ denotes the Euclidean normal vector field of $\mathcal{M}$. Thus a simple calculation reveals that for a strictly convex and $C^{2}$-bounded $M$, the corresponding hypersurface $\mathcal{M}$ is strictly convex and bounded in $C^{2}$, as long as $M$ stays away from the point $r=0$ and its antipodal point.

The closure of a weakly convex set is again a weakly convex set. However, this statement is not true for convex sets (neither in spheres nor in hemispheres). We want to prove a sufficient condition for a weakly convex body in a hemisphere to be a convex body in a hemisphere.

THEOREM 3.5. Let $n \geq 1$ and $\hat{M} \subset \mathbb{S}^{n+1}$ be a weakly convex body in a hemisphere $\mathcal{H}\left(x_{0}\right)$ for some $x_{0} \in \mathbb{S}^{n+1}$. Suppose that $\hat{M}$ satisfies an interior sphere condition with respect to $x_{0}$ at all points $p \in \hat{M} \cap \mathcal{S}\left(x_{0}\right)$. Then $\hat{M}$ is a convex body in a hemisphere.

Firstly we need some lemmata.

LEMma 3.6. Let $n \geq 1$ and $x_{0} \in \mathbb{S}^{n+1}$. Let $p \in \mathcal{S}\left(x_{0}\right)$. Let $\gamma:[0, \pi] \rightarrow \mathbb{S}^{n+1}$ be a $C^{1}$-geodesic, parametrized by arc length, with $\gamma(0)=p$. Let $\tilde{p}$ denote the outward normal vector of $\mathcal{H}\left(x_{0}\right)$ at $p$. Then

$$
\langle\dot{\gamma}(0), \tilde{p}\rangle<(>,=),
$$

if and only if the geodesic satisfies $\gamma(t) \in \operatorname{int} \mathcal{H}\left(x_{0}\right)\left(\mathcal{C H}\left(x_{0}\right), \mathcal{S}\left(x_{0}\right)\right)$ for some (and hence every) $t \in(0, \pi)$.

Proof. First of all we note the following fact: Since $\gamma$ is a $C^{1}$-geodesic, $\gamma$ is a segment of a great circle. Hence a third point lying on $\gamma$ determines uniquely the great circle $\Gamma$ such that $\gamma([0, \pi]) \subset \Gamma$. Thus the existence of $t \in(0, \pi)$, such that $\gamma(t) \in \operatorname{int} \mathcal{H}\left(x_{0}\right)\left(\mathcal{C H}\left(x_{0}\right), \mathcal{S}\left(x_{0}\right)\right)$ implies $\gamma((0, \pi)) \subset \operatorname{int} \mathcal{H}\left(x_{0}\right)\left(\complement \mathcal{H}\left(x_{0}\right), \mathcal{S}\left(x_{0}\right)\right)$.

Suppose firstly that $\langle\dot{\gamma}(0), \tilde{p}\rangle<0$. Then $\gamma(t) \in \operatorname{int} \mathcal{H}\left(x_{0}\right)$ for $t$ close to 0 . Hence by the observation made above, we obtain $\gamma((0, \pi)) \subset \operatorname{int} \mathcal{H}\left(x_{0}\right)$.

If on the other hand there exists $t \in(0, \pi)$, such that $\gamma(t) \in \operatorname{int} \mathcal{H}\left(x_{0}\right)$, then the geodesic $\tilde{\gamma}:[0, t] \rightarrow \mathbb{S}^{n+1}$ with $\tilde{\gamma}(0)=\gamma(t)$ and $\dot{\tilde{\gamma}}(s)=-\dot{\gamma}(t-s)$ satisfies $\langle\dot{\tilde{\gamma}}(t), \tilde{p}\rangle>0$, hence we obtain $\langle\dot{\gamma}(0), \tilde{p}\rangle<0$.

LEMma 3.7. Let $n \geq 1$ and $\hat{M} \subset \mathbb{S}^{n+1}$ be a weakly convex body in the hemisphere $\mathcal{H}\left(x_{0}\right)$ for some fixed $x_{0} \in \mathbb{S}^{n+1}$. Let $p \in \hat{M} \cap \mathcal{S}\left(x_{0}\right)$ and suppose that $\hat{M}$ satisfies an interior sphere condition at $p$ with respect to $x_{0}$. Let $\gamma \in C^{1}\left(\left[0, t_{0}\right), \mathbb{S}^{n+1}\right), 0<t_{0}$, be a geodesic with $\gamma(0)=p$ and $\gamma\left(\left(0, t_{0}\right)\right) \subset$ int $\mathcal{H}\left(x_{0}\right)$. Then there exists $0<\delta \leq t_{0}$, such that $\gamma((0, \delta)) \subset \operatorname{int} \hat{M}$.

Proof. Let $\tilde{p}$ denote the outward normal vector of $\mathcal{H}\left(x_{0}\right)$ at $p$. From Lemma 3.6 we obtain

$$
\langle\dot{\gamma}(0), \tilde{p}\rangle<0 .
$$

Let us look at the situation in the coordinates given by the stereographic projection $\mathcal{P}$ yielding the metric (3.3). Let $\rho>0$ and $B_{\rho}(\bar{p})$ be an inball with respect to $\mathcal{P}(p)$ with center $\bar{p}$. Let $\tilde{\gamma}:=\mathcal{P} \circ \gamma$, then since the metric of the sphere is conformally 
equivalent to the Euclidean metric in stereographic coordinates, we obtain from (3.6) that

$$
\langle\dot{\tilde{\gamma}}(0), \nu(\mathcal{P}(p))\rangle<0
$$

where $\nu(\mathcal{P}(p))$ denotes the outward normal of $B_{2}(0)$ at $\mathcal{P}(p)$. Since the inball $B_{\rho}(\bar{p})$ is tangent to $\partial B_{2}(0)$, we obtain some small $\delta>0$, such that for $t \in(0, \delta)$ :

$$
\tilde{\gamma}(t) \in B_{\rho}(\bar{p})
$$

Proof of Theorem 3.5. Let $p, q \in \hat{M}$ be two arbitrary points, then we have to show that an arbitrary minimizing geodesic $\hat{\gamma}_{p, q}$ connecting $p$ and $q$ and contained in $\mathcal{H}\left(x_{0}\right)$ is contained in $\hat{M}$.

If no antipodal points exist in $\hat{M}$, we have nothing to prove. Hence let us assume that there exist points $p, q \in \hat{M}$ with $\operatorname{dist}(p, q)=\pi$. We will show that then $\hat{M}=$ $\mathcal{H}(x)$.

Since $\hat{M} \subset \mathcal{H}(x)$ we know that $p, q \in \mathcal{S}(x)$. Let $y \in \operatorname{int} \mathcal{H}(x)$ be arbitrary. Then there exists a unique $C^{1}$-geodesic $\gamma:[0, \pi] \rightarrow \mathbb{S}^{n+1}$ starting at $p$ and ending at $q$, such that $y \in \gamma((0, \pi)) \subset \operatorname{int} \mathcal{H}(x)$. From Lemma 3.7 applied to $p$ and $q$ we obtain that $\gamma((0, \pi)) \subset \hat{M}$. Hence int $\mathcal{H}(x) \subset \hat{M}$ and we infer $\hat{M}=\overline{\hat{M}}=\mathcal{H}(x)$.

Hence we know that the weakly convex body $\hat{M}$ in Theorem 1.1 is a convex body in a hemisphere. Thus it remains to distinguish two cases: $\hat{M}$ does or does not contain a pair of antipodal points.

The following lemma is standard and a proof can be found in $[6$, Chapter 3 , Corollary 1].

LemmA 3.8. Let $n \geq 1$ and $\hat{M} \subset \mathbb{S}^{n+1}$ be a convex body in the sphere, which does not contain any antipodal points. Then $\hat{M}$ is contained in an open hemisphere.

Proof of Theorem 1.1. From Theorem 3.5 we obtain that $\hat{M}$ is a convex body in a hemisphere. Hence if $\hat{M}$ contains a pair of antipodal points, then $\hat{M}=\mathcal{H}\left(x_{0}\right)$. Otherwise it is contained in an open hemisphere in view of Lemma 3.8.

For $C^{2}$-hypersurfaces we obtain a generalization of Theorem 1, see Corollary 1.2. For the proof of this result, we need one further Lemma.

LEMMA 3.9. Let $\hat{M}_{n} \subset \mathcal{H}\left(x_{n}\right) \subset \mathbb{S}^{n+1}$ be a sequence of sets, such that

$$
\hat{M}_{n} \subset \operatorname{int} \hat{M}_{n+1},
$$

then there exists $x_{0} \in \mathbb{S}^{n+1}$, such that

$$
\hat{M}_{n} \subset \operatorname{int} \mathcal{H}\left(x_{0}\right) \quad \forall n \in \mathbb{N} .
$$

Proof. A subsequence of points $x_{n_{k}}$ converges to some $x_{0} \in \mathbb{S}^{n+1}$. We claim that this $x_{0}$ is a point which satisfies (3.10). If this was not the case, then we use the monotonicity to derive the existence of $n_{0} \in \mathbb{N}$ with the property

$$
\hat{M}_{n_{0}} \cap \mathcal{H}\left(x_{0}\right)^{c} \neq \emptyset .
$$


Thus there exists a point

$$
y \in \hat{M}_{n}, n \geq n_{0}
$$

and $\epsilon>0$ with the property

$$
\operatorname{dist}\left(y, \mathcal{H}\left(x_{0}\right)\right) \geq \epsilon
$$

This leads to a contradiction, since for large $k$ we have

$$
y \in \hat{M}_{n_{k}-1} \subset \operatorname{int} \mathcal{H}\left(x_{n_{k}}\right) \subset \operatorname{int} \mathcal{H}\left(x_{n_{k}}\right) \cup \operatorname{int} \mathcal{H}\left(x_{0}\right)
$$

and the maximal distance of points in the latter set to $x_{0}$ converges to $\frac{\pi}{2}$.

Proof of Corollary 1.2. Choose a differentiable normal vector field $\nu$, such that the second fundamental form with respect to $-\nu$ is positive semi-definite and let $\mathcal{U}$ be a tubular neighborhood around $M$ with corresponding signed distance function $d \in C^{2}(\mathcal{U})$ and normal Gaussian coordinate system $\left(x^{\alpha}\right)$, compare [10, Theorem 1.3.13]. Note that $d=x^{0}$ and $\nabla d=\nu$. According to [10, Lemma 2.4.3], the second fundamental form with respect to $-\nu$ of the coordinate slices

$$
\left\{x^{0}=\text { const }\right\},
$$

which can be seen as a solution to the flow

$$
\dot{x}=\nu, x(t, \xi)=(t, \xi),
$$

evolves according to the evolution equation

$$
\dot{h}_{j}^{i}=-h_{j}^{k} h_{k}^{i}-\delta_{j}^{i} .
$$

Thus the principle curvatures of the slices are strictly decreasing, which implies, that the hypersurfaces

$$
M_{-t}=\left\{x^{0}=-t\right\}
$$

are strictly convex with positive definite second fundamental form. Consider the image $\mathcal{M}_{-t}$ under a suitable stereographic projection $\mathcal{P}$, which is a strictly convex $C^{2}$ hypersurface in $\mathbb{R}^{n+1}$. For any small $\delta>0$ there exists $\epsilon(\delta)>0$, such that for the convolution $d_{\epsilon}$ of the signed distance function there hold

$$
\begin{gathered}
\mathcal{M}_{-t}^{\epsilon} \equiv\left\{d_{\epsilon}=-\left(t+\frac{\delta}{2}\right)\right\} \subset \mathcal{P}(\{-(t+\delta)<d<-t\}), \\
\left\langle\nabla d_{\epsilon}, \nabla d\right\rangle \geq c>0
\end{gathered}
$$

and

$$
\mathcal{M}_{-t}^{\epsilon} \text { is strictly convex. }
$$

Using (3.4) and the $C^{2}$-convergence of the convolution, those properties carry over to $M_{-t}^{\epsilon} \equiv \mathcal{P}^{-1}\left(\mathcal{M}_{-t}^{\epsilon}\right)$, a hypersurface in $\mathbb{S}^{n+1}$, to which we may apply do Carmo's and Warner's theorem. Using the same construction, we obtain

$$
M_{-\frac{t}{2}}^{\epsilon_{1}} \text {, where } \epsilon_{1}=\epsilon\left(\frac{t}{2}\right) \text {. }
$$


Thus we derive a sequence of smooth and strictly convex hypersurfaces

$$
M_{n} \equiv M_{-\frac{t}{2^{n}}}^{\epsilon_{n}}, \text { where } \epsilon_{n}=\epsilon\left(\frac{t}{2^{n}}\right)
$$

with the property

$$
M_{n} \subset \operatorname{int} \hat{M}_{n+1}
$$

Here we also used the generalized Jordan curve theorem, cf. [3, Chapter IV, 19]. Lemma 3.9 implies that there exists $x_{0} \in \mathbb{S}^{n+1}$ such that $M \subset \mathcal{H}\left(x_{0}\right)$ and Theorem 3.5 shows that $M$ bounds a convex body $\hat{M}=\overline{\bigcup_{n \in \mathbb{N}} \hat{M}_{n}}$ in a hemisphere, since $\hat{M}$ obviously satisfies the interior sphere condition with respect to $x_{0}$ at all points of $\hat{M} \cap \mathcal{S}\left(x_{0}\right)$.

\section{The curvature flow and first estimates.}

Curvature functions. Now we mention some elementary facts about curvature functions on a hypersurface.

To derive the geometric inequalities, we will need some properties of the elementary symmetric polynomials.

LEMMA 4.1. Let $1 \leq k \leq n$ be fixed.

(i) We define the convex cone

$$
\Gamma_{k}=\left\{\left(\kappa_{i}\right) \in \mathbb{R}^{n}: H_{1}\left(\kappa_{i}\right)>0, H_{2}\left(\kappa_{i}\right)>0, \ldots, H_{k}\left(\kappa_{i}\right)>0\right\} .
$$

Then $H_{k}$ is strictly monotone on $\Gamma_{k}$ and $\Gamma_{k}$ is exactly the connected component of

$$
\left\{\left(\kappa_{i}\right) \in \mathbb{R}^{n}: H_{k}\left(\kappa_{i}\right)>0\right\}
$$

containing the positive cone.

(ii) The $k$-th roots $\sigma_{k}=H_{k}^{\frac{1}{k}}$ are concave on $\Gamma_{k}$.

(iii) For $1<s<t<n$ and $\tilde{\sigma}_{k}=\left(\frac{H_{k}}{\left(\begin{array}{c}n \\ k\end{array}\right)}\right)^{\frac{1}{k}}$ there holds

$$
\tilde{\sigma}_{n} \leq \tilde{\sigma}_{t} \leq \tilde{\sigma}_{s} \leq \tilde{\sigma}_{1},
$$

where the principal curvatures have to lie in $\Gamma_{n} \equiv \Gamma_{+}$for the first, in $\Gamma_{t}$ for the second and in $\Gamma_{s}$ for the third inequality.

(iv) For fixed $i$, no summation over $i$, there holds

$$
H_{k}=\frac{\partial H_{k+1}}{\partial \kappa_{i}}+\kappa_{i} \frac{\partial H_{k}}{\partial \kappa_{i}}
$$

Proof. The convexity of the cone $\Gamma_{k}$ and (i) follows from [18, Section 2], (ii) from [14, Thm. 3.2], (iii) from [20, Lemma 15.12] and (iv) follows directly from the definition of the $H_{k}$.

A consequence of the preceding lemma is the following

LEMma 4.2. Let $N$ be a semi-Riemannian space of constant curvature, then the symmetric polynomials $F=H_{k}, 1 \leq k \leq n$, are divergence free for every admissible 
hypersurface $M$ of $N$. In case $k=2$ it suffices to assume that $N$ is an Einstein manifold.

Proof. The proof of the lemma can be found in [11, Lemma 5.8]. The proof consists of induction on $k$ and (iv) of Lemma 4.1.

Now we state a well-known inequality for general curvature functions:

LEMma 4.3. Let $F \in C^{2}\left(\Gamma_{+}\right) \cap C^{0}\left(\bar{\Gamma}_{+}\right)$be a strictly monotone, concave curvature function, positively homogeneous of degree 1 with $F(1, \ldots, 1)>0$, then

$$
F(\kappa) \leq \frac{F(1, \ldots, 1)}{n} H(\kappa)
$$

and

$$
\sum_{i=1}^{n} F_{i}(\kappa) \geq F(1, \ldots, 1)
$$

where $\kappa=\left(\kappa_{k}\right) \in \Gamma_{+}$.

Proof. See [10, Lemma 2.2.19, Lemma 2.2.20].

Evolution equations for the curvature flow. The following evolution equations are valid for curvature flows in the sphere, for a derivation see [10, Chapter $2]$.

LEMma 4.4. (Evolution equations)

$$
\begin{gathered}
\frac{d}{d t} g_{i j}=-2 \Phi h_{i j} \\
\frac{d}{d t} h_{i j}-\Phi^{\prime} F^{k l} h_{i j ; k l}=\Phi^{\prime} F^{k l} h_{r k} h_{l}^{r} h_{i j}-\left(\Phi^{\prime} F+\Phi\right) h_{r i} h_{j}^{r} \\
+\left(\Phi^{\prime} F+\Phi\right) g_{i j}-\Phi^{\prime} F^{k l} g_{k l} h_{i j}+\Phi^{k l, r s} h_{k l ; i} h_{r s ; j} . \\
\frac{d}{d t} h_{j}^{i}-\Phi^{\prime} F^{k l} h_{j ; k l}^{i}=\Phi^{\prime} F^{k l} h_{k}^{r} h_{r l} h_{j}^{i}+\left(\Phi-\Phi^{\prime} F\right) h_{k}^{i} h_{j}^{k} \\
+\Phi^{\prime} F^{k l, r s} h_{k l ; m} h_{r s ; j} g^{m i}+\Phi^{\prime \prime} F^{i} F_{j} \\
+\left(\Phi+\Phi^{\prime} F\right) \delta_{j}^{i}-\Phi^{\prime} F^{k l} g_{k l} h_{j}^{i} . \\
\frac{d}{d t} \Phi-\Phi^{\prime} F^{k l} \Phi_{; k l}=\Phi^{\prime} F^{k l} h_{k r} h_{l}^{r} \Phi+\Phi^{\prime} F^{k l} g_{k l} \Phi
\end{gathered}
$$

Curvature estimates. Since the flow reduces to a scalar parabolic equation, see for example [10, Chapter 2.5], short-time existence is guaranteed and we know that the flow exists on a maximal time interval $\left[0, T^{*}\right)$ for some $0<T^{*} \leq \infty$. Firstly, we show that the flow exists only up to a finite time $T^{*}$.

LEMMA 4.5. There holds

$$
T^{*}<\infty
$$


Proof. Using Lemma 4.3 we can compare the evolution of $-\Phi$, see (4.10), with the solution of the ordinary differential equation

$$
\dot{\varphi}=\operatorname{pn} \varphi^{\frac{2 p+1}{p}} \text {. }
$$

This shows that $\sup _{x \in M_{t}}(-\Phi(x))$ becomes unbounded in finite time.

We show that the hypersurfaces remain strictly convex and the principal curvatures are uniformly bounded from above. For curvature functions with $F_{\mid \partial \Gamma_{+}}=0$ the strict convexity of the flow hypersurfaces follows immediately. For concave and inverse concave curvature functions this follows from the following.

LEMma 4.6. Suppose that $F$ is a curvature function as in 1.3 (i) with $p=1$. Then there exists a constant $c>0$, such that the principal curvatures of the flow $\kappa_{1}, \ldots, \kappa_{n}$ satisfy

$$
\kappa_{n} \leq c \kappa_{1} .
$$

Proof. Let $0<\epsilon<\frac{1}{n}$, such that

$$
T_{i j}=h_{i j}-\epsilon H g_{i j}
$$

is positive definite at $t=0 . T_{i j}$ satisfies

$$
\begin{aligned}
T_{i j}-\Phi^{\prime} F^{k l} T_{i j ; k l}= & \Phi^{\prime} F^{k l} h_{r k} h_{l}^{r} T_{i j}-\Phi^{\prime} F^{k l} g_{k l} T_{i j} \\
& +\epsilon\left(\Phi^{\prime} F-\Phi\right)\|A\|^{2} g_{i j}+2 \epsilon \Phi H h_{i j} \\
& +\Phi^{k l, r s} h_{k l ; i} h_{r s ; j}-\epsilon \Phi^{k l, r s} h_{k l ; m} h_{r s ;}{ }^{m} g_{i j} \\
\equiv & N_{i j}+\tilde{N}_{i j},
\end{aligned}
$$

where we also used $\Phi^{\prime} F+\Phi=0$, due to $p=1$. Here $\tilde{N}_{i j}$ denotes the terms involving derivatives of $h_{i j}$. At a point $\left(t_{0}, \xi_{0}\right)$ let $\eta$ be a null-eigenvector of $T_{i j}$, i.e.

$$
h_{i j} \eta^{j}=\epsilon H \eta_{i}
$$

and $T_{i j} \geq 0$ elsewhere. There holds

$$
\begin{aligned}
N_{i j} \eta^{i} \eta^{j} & =2 \epsilon \Phi^{\prime} F\|A\|^{2}\|\eta\|^{2}+2 \epsilon^{2} \Phi H^{2}\|\eta\|^{2} \\
& \geq 2 \epsilon \Phi^{\prime} F H^{2}\|\eta\|^{2}\left(\frac{1}{n}-\epsilon\right)>0 .
\end{aligned}
$$

To prove that $\tilde{N}_{i j}$ satisfies a modified null-eigenvector condition, note that $\Phi$ is, as a function of the principal curvatures, symmetric, monotone, concave and inverse concave in the sense of [2, Sec. 2]. We apply [2, Theorem 4.1] to obtain

$$
\tilde{N}_{i j} \eta^{i} \eta^{j}+2 \sup _{\Gamma} \Phi^{k l}\left(2 \Gamma_{k}^{r}\left(h_{r i ; l} \eta^{i}-\epsilon H_{l} \eta_{r}-\Gamma_{k}^{r} \Gamma_{l}^{q}\left(h_{r q}-\epsilon H \delta_{r q}\right)\right)\right) \geq 0 .
$$

Those are exactly the requirements to apply Andrews' generalized maximum principle, $\left[2\right.$, Theorem 3.2] to conclude $T_{i j}>0$ for all $t \in\left[0, T^{*}\right)$.

Next we derive upper bounds for the principal curvatures. 
Lemma 4.7. There holds

$$
\sup _{t \in\left[0, T^{*}\right), x \in M_{t}} H(x) \leq \sup _{M_{0}} H
$$

Proof. Let $0<T<T^{*}$ be arbitrary. Suppose there exists $t_{0} \in(0, T]$ and $x_{0} \in M_{t_{0}}$ such that

$$
\sup _{t \in\left[0, t_{0}\right], x \in M_{t}} H \leq H\left(x_{0}\right) .
$$

Then we obtain from the maximum principle and the concavity of $F$, that at $x_{0}$ there holds in view of $(4.9)$

$$
\begin{aligned}
0 \leq & p F^{k l} h_{k}^{r} h_{r l} \frac{H}{F^{p+1}}-(p+1) \frac{|A|^{2}}{F^{p}}-p F^{k l} g_{k l} \frac{H}{F^{p+1}}-(1-p) \frac{n}{F^{p}} \\
= & \frac{p}{F^{p+1}}\left(F^{k l} h_{k}^{r} h_{r l} H-F|A|^{2}\right)-\frac{n+|A|^{2}}{F^{p}} \\
& -\frac{p}{F^{p+1}}\left(F^{k l} g_{k l} H-n F\right) .
\end{aligned}
$$

However, we note that

$$
\begin{aligned}
F^{k l} h_{k}^{r} h_{r l} H-F|A|^{2} & =\sum_{i, j}\left(f_{i} \kappa_{i}^{2} \kappa_{j}-f_{i} \kappa_{i} \kappa_{j}^{2}\right) \\
& =\sum_{i<j} \kappa_{i} \kappa_{j}\left(\kappa_{i}-\kappa_{j}\right)\left(f_{i}-f_{j}\right) \leq 0,
\end{aligned}
$$

since for concave curvature functions there holds $f_{i} \geq f_{j}$ for $i<j$. Furthermore, in view of Lemma 4.3 we obtain

$$
F^{k l} g_{k l} H-n F \geq 0
$$

Hence we obtain a contradiction.

\section{Elementary flow properties and further curvature estimates.}

Lemma 5.1. Let $M_{t}, 0 \leq t<T^{*}$, be a flow hypersurface and $\hat{M}_{t}$ be the enclosed convex body, cf. Corollary 1.2. Then those convex bodies are strictly monotonically ordered, i.e.

$$
s<t \Rightarrow \hat{M}_{s} \subset \operatorname{int} \hat{M}_{t}
$$

Proof. Let $0 \leq s<T^{*}$. Then the flow hypersurface $M_{s}$ is strictly convex, as was shown in the previous section. Thus, using Corollary 1.2, we first conclude that $M_{s}$ does indeed enclose a convex body $\hat{M}_{s}$ and that this body has to lie compactly in an open hemisphere $\operatorname{int} \mathcal{H}\left(\tilde{x}_{s}\right)$. Choose $x_{s} \in \operatorname{int} \hat{M}_{s}$. Thus $M_{s}$, which in particular is starshaped with respect to $x_{s}$, can be written as a graph over $\mathcal{S}\left(x_{s}\right)$,

$$
M_{s}=\operatorname{graph} u\left(s, \mathcal{S}\left(x_{s}\right)\right),
$$

and thus there is an $\epsilon>0$, such that for all $s \leq t<s+\epsilon$ the hypersurfaces $M_{t}$ may be written as a graph over $S\left(x_{s}\right)$, compare [10, Theorem 2.5.19]. In these coordinates $u$ locally satisfies the scalar flow equation

$$
\frac{\partial u}{\partial t}=\frac{v}{F^{p}}
$$


cf. [10, p. 98-99], and thus the function $u$ is strictly increasing for fixed $x \in \mathcal{S}\left(x_{s}\right)$. Since

$$
\hat{M}_{s}=\left\{\left(r,\left(x^{i}\right)\right) \in \mathbb{R} \times \mathcal{S}\left(x_{s}\right): 0 \leq r \leq u\left(s,\left(x^{i}\right)\right)\right\},
$$

where $\left(r,\left(x^{i}\right)\right)$ describe the corresponding geodesic polar coordinates around $x_{s}$, the claim follows.

Proposition 5.2. There is a uniquely determined limit surface $M_{T^{*}}$, which can be written as a graph in geodesic polar coordinates,

$$
M_{T^{*}}=\operatorname{graph} u\left(T^{*}, \mathcal{S}\left(y_{0}\right)\right),
$$

where $y_{0} \in \operatorname{int} \hat{M}_{T^{*}}$. Furthermore there holds

$$
u(t, \cdot) \rightarrow u\left(T^{*}, \cdot\right) \quad \text { in } C^{1, \beta}\left(\mathcal{S}\left(y_{0}\right)\right) \quad \forall 0 \leq \beta<1 .
$$

Proof. In geodesic polar coordinates the metric of $\mathbb{S}^{n+1}$ is given by

$$
d \bar{s}^{2}=d r^{2}+\sin ^{2} r \sigma_{i j} d x^{i} d x^{j}, 0<r<\pi .
$$

Let $y_{0} \in \operatorname{int} \hat{M}_{0}$ and $\hat{y}_{0}$ denote the antipodal point of $y_{0}$, then by Lemma 3.9 we know that

$$
\hat{M}_{t} \subset K \Subset \mathbb{S}^{n+1} \backslash\left\{\hat{y}_{0}\right\} \quad \forall 0 \leq t<T^{*} .
$$

Thus we have a uniform parametrization of the flow hypersurfaces as graphs over $\mathcal{S}\left(y_{0}\right)$

$$
M_{t}=\operatorname{graph} u(t, \cdot) .
$$

The quantity $v^{2}=1+\sin ^{-2} u \sigma^{i j} u_{i} u_{j}$ is bounded by convexity, see [10, Theorem 2.7.10]. The second fundamental form of a graph hypersurface satisfies

$$
h_{j}^{i}=\frac{\dot{\vartheta}}{v \vartheta} \delta_{j}^{i}+\frac{\dot{\vartheta}}{v^{3} \vartheta^{3}} u^{i} u_{j}-\frac{\tilde{\sigma}^{i k}}{v \vartheta^{2}} u_{k j},
$$

where $\vartheta=\sin u$ and $\tilde{\sigma}^{i k}$ is the inverse of

$$
\tilde{\sigma}_{i k}=\varphi_{i} \varphi_{k}+\sigma_{i k}, \quad \varphi=\int_{r_{0}}^{u} \sin ^{-1}(s) d s,
$$

cf. [25, (3.82)]. Here covariant differentiation and index raising is performed with respect to the metric $\sigma_{i j}$ and by Lemma 4.7 we obtain uniform $C^{2}$ estimates for the $u(t, \cdot)$. This gives the existence of a convergent subsequence with uniquely determined $C^{1}$ limit $u\left(T^{*}, \cdot\right)$, using monotonicity.

Proposition 5.3. Suppose that on some time interval $[s, t] \subset\left(0, T^{*}\right)$ there is a point $x_{0} \in \operatorname{int} \hat{M}_{s}$, such that for a common parametrization of the surfaces $M_{\tau}$,

$$
M_{\tau}=\operatorname{graph} u\left(\tau, \mathcal{S}\left(x_{0}\right)\right),
$$

there is a constant $\epsilon>0$ satisfying $u \leq \frac{\pi}{2}-\epsilon$, then the curvature function of those hypersurfaces is uniformly positive,

$$
F \geq \tilde{c}>0,
$$


where $\tilde{c}$ depends on $\epsilon$.

Proof. Using [10, Lemma 3.3.2] and (4.10) we deduce the evolution equations for $-\Phi$ and $u$ to be

$$
\frac{d}{d t}(-\Phi)-\Phi^{\prime} F^{i j}(-\Phi)_{i j}=\Phi^{\prime} F^{i j} h_{i k} h_{j}^{k}(-\Phi)+\Phi^{\prime} F^{i j} g_{i j}(-\Phi)
$$

and

$$
\frac{d}{d t} u-\Phi^{\prime} F^{i j} u_{i j}=\left(p^{-1}+1\right) \Phi^{\prime} F v^{-1}-\Phi^{\prime} F^{i j} \bar{h}_{i j}
$$

Set

$$
w=\log (-\Phi)+f(u)
$$

where $f$ will be specified later. Then

$$
\begin{aligned}
\frac{d}{d t} w-\Phi^{\prime} F^{i j} w_{i j}= & \Phi^{\prime} F^{i j} h_{i k} h_{j}^{k}+\Phi^{\prime} F^{i j} g_{i j}+\Phi^{\prime} F^{i j} \frac{\Phi_{i}}{\Phi} \frac{\Phi_{j}}{\Phi} \\
& +\left(p^{-1}+1\right) f^{\prime} \Phi^{\prime} F v^{-1}-f^{\prime} \Phi^{\prime} F^{i j} \bar{h}_{i j}-f^{\prime \prime} \Phi^{\prime} F^{i j} u_{i} u_{j}
\end{aligned}
$$

We want to bound the function $w$. Thus suppose without loss of generality, that

$$
\sup _{(\tau, \xi) \in(s, t] \times M} w(\tau, \xi)=w\left(t_{0}, \xi_{0}\right) .
$$

Then at this point we have

$$
\frac{\Phi_{i}}{\Phi}=-f^{\prime}(u) u_{i}
$$

and thus, also using $\bar{h}_{i j}=\frac{\bar{H}}{n} \bar{g}_{i j}=\frac{\bar{H}}{n} g_{i j}-\frac{\bar{H}}{n} u_{i} u_{j}$, we find at $\left(t_{0}, \xi_{0}\right)$ that

$$
\begin{aligned}
0 \leq & \Phi^{\prime} F^{i j} h_{i k} h_{j}^{k}+\left(1-f^{\prime} \frac{\bar{H}}{n}\right) \Phi^{\prime} F^{i j} g_{i j}+\left(p^{-1}+1\right) f^{\prime} \Phi^{\prime} F v^{-1} \\
& +\left(\left(f^{\prime}\right)^{2}+f^{\prime} \frac{\bar{H}}{n}-f^{\prime \prime}\right) \Phi^{\prime} F^{i j} u_{i} u_{j} .
\end{aligned}
$$

Now define the function

$$
f(u)=-\log \left(\cos u-\cos \left(\frac{\pi}{2}-\frac{\epsilon}{2}\right)\right)=-\log (\cos u-c),
$$

where $c:=\cos \left(\frac{\pi}{2}-\frac{\epsilon}{2}\right)$, such that by assumption $f$ is strictly positive and uniformly bounded for $\tau \in[s, t]$. We have

$$
f^{\prime}=\frac{\sin u}{\cos u-c}
$$

and

$$
f^{\prime \prime}=\frac{1}{(\cos u-c)^{2}}-\frac{c \cdot \cos u}{(\cos u-c)^{2}}
$$


and thus in view of $(2.14)$

$$
f^{\prime} \frac{\bar{H}}{n}=\frac{\cos u}{\cos u-c}>1+\delta, \delta=\delta(\epsilon),
$$

and

$$
\left(f^{\prime}\right)^{2}+f^{\prime} \frac{\bar{H}}{n}-f^{\prime \prime}=0 .
$$

Since $F^{i j} h_{i k} h_{j}^{k} \leq F H$ due to the convexity of the hypersurfaces, we conclude at $\left(t_{0}, \xi_{0}\right)$

$$
\begin{aligned}
0 & \leq \Phi^{\prime} F^{i j} h_{i k} h_{j}^{k}+\left(p^{-1}+1\right) f^{\prime} \Phi^{\prime} F v^{-1}-n \delta \Phi^{\prime} \\
& \leq \frac{p}{F^{p}} H+(p+1) \frac{f^{\prime}}{v F^{p}}-n \delta \frac{p}{F^{p+1}} .
\end{aligned}
$$

Supposing that $w\left(t_{0}, \xi_{0}\right)$ is very large, $-\Phi$ must also be very large, which leads to a contradiction, since $H$ is bounded by Lemma 4.7. Hence $w$, and thus also $-\Phi$, must be bounded.

Now we characterize $T^{*}$, where in particular we show, that the flow exists as long as the hypersurfaces remain strictly convex.

Proposition 5.4. Suppose that on some interval $[s, t) \subset\left[0, T^{*}\right)$ we have

$$
F(\tau, \xi) \geq \epsilon>0 \quad \forall(\tau, \xi) \in[s, t) \times \mathbb{S}^{n}
$$

then there holds

$$
T^{*}>t
$$

Proof. Without loss of generality suppose that all $M_{\tau}, s \leq \tau<t$, are uniformly parametrized over $\mathcal{S}\left(y_{0}\right)$. The second fundamental form has, with respect to the corresponding spherical metric, the form

$$
h_{j}^{i}=\frac{\dot{\vartheta}}{v \vartheta} \delta_{j}^{i}+\frac{\dot{\vartheta}}{v^{3} \vartheta^{3}} u^{i} u_{j}-\frac{\tilde{\sigma}^{i k}}{v \vartheta^{2}} u_{k j}
$$

The scalar flow equation is

$$
\frac{\partial}{\partial t} u=\frac{v}{F^{p}\left(h_{j}^{i}\right)} \equiv G\left(x, u, D u, D^{2} u\right)
$$

Thus

$$
\frac{\partial G}{\partial u_{i j}}=-\frac{p v}{F^{p+1}} F_{l}^{k} \frac{\partial h_{k}^{l}}{\partial u_{i j}}=\frac{p v}{F^{p+1}} F_{l}^{k} \frac{\tilde{\sigma}^{l m}}{v \vartheta^{2}} \delta_{m}^{i} \delta_{k}^{j}=\frac{p}{\vartheta^{2} F^{p+1}} F_{l}^{j} \tilde{\sigma}^{l i},
$$

where the latter is uniformly positive definite by assumption, as well as by Lemma 4.6 or $\left.F\right|_{\partial \Gamma_{+}}=0$. Then, using the regularity results of Krylov and Safonov, cf. [19], and Schauder theory, we obtain uniform $C^{4, \alpha}$ estimates on $[s, t)$ and thus the flow extends beyond $t$. 
6. Convergence to an equator. In view of Lemma 5.1 we know that there exists $x_{0} \in \mathbb{S}^{n+1}$, such that $\hat{M}_{t} \subset \operatorname{int} \mathcal{H}\left(x_{0}\right)$. Now we want to show, that the limit hypersurface $M_{T^{*}}$ is equal to $\mathcal{S}\left(x_{0}\right)$. We first view the hypersurfaces as embedded in Euclidean space using the conformally flat parametrization of $\mathbb{S}^{n+1}$.

LEMma 6.1. The enclosed, weakly convex body of $M_{T^{*}}, \hat{M}_{T^{*}}$, satisfies a uniform interior sphere condition.

Proof. We will denote $\mathcal{P}_{x_{0}}\left(M_{t}\right)$ by $\mathcal{M}_{t}$ for $t \in\left[0, T^{*}\right]$. Since all $M_{t}$ range within distance less than $\frac{\pi}{2}$ around $x_{0}$, the metrics $\bar{g}_{\alpha \beta}$ and $\hat{g}_{\alpha \beta}$ are uniformly equivalent on the set of consideration. Thus we also obtain the $C^{1}$-convergence of $\mathcal{M}_{t} \rightarrow \mathcal{M}_{T^{*}}$. Let $x \in \mathcal{M}_{T^{*}}$ be arbitrary and $t_{n}, x_{n} \in \mathcal{M}_{t_{n}}$ be sequences such that

$$
t_{n} \rightarrow T^{*}, x_{n} \rightarrow x
$$

By Remark 3.4 we obtain a sequence of inballs with center $y_{n} \in \operatorname{int} \hat{\mathcal{M}}_{t_{n}}$ and uniform radius $R$, such that

$$
B_{R}\left(y_{n}\right) \subset \operatorname{int} \hat{\mathcal{M}}_{t_{n}} \wedge \partial B_{R}\left(y_{n}\right) \cap \mathcal{M}_{t_{n}}=\left\{x_{n}\right\} .
$$

Without loss of generality we have

$$
y_{n} \rightarrow y \in \operatorname{int} \hat{\mathcal{M}}_{T^{*}}
$$

First of all, let $z \in B_{R}(y)$. By the triangle inequality, for large $n$ there holds

$$
z \in B_{R}\left(y_{n}\right) \subset \operatorname{int} \hat{\mathcal{M}}_{t_{n}} \subset \operatorname{int} \hat{\mathcal{M}}_{T^{*}}
$$

Thus

$$
B_{R}(y) \subset \operatorname{int} \hat{\mathcal{M}}_{T^{*}}
$$

There holds

$$
\operatorname{dist}(x, y) \leq \operatorname{dist}\left(x, x_{n}\right)+\operatorname{dist}\left(x_{n}, y_{n}\right)+\operatorname{dist}\left(y_{n}, y\right) \rightarrow R
$$

and thus

$$
\operatorname{dist}(x, y)=R,
$$

since $x \in \mathcal{M}_{T^{*}}$. We summarize:

$$
B_{R}(y) \subset \operatorname{int} \hat{\mathcal{M}}_{T^{*}} \wedge\{x\} \subset \partial B_{R}(y) \cap \mathcal{M}_{T^{*}} .
$$

If we now choose an inball

$$
B_{\frac{R}{2}}(\tilde{y}) \subset B_{R}(y)
$$

with the property

$$
\partial B_{\frac{R}{2}}(\tilde{y}) \cap \partial B_{R}(y)=\{x\},
$$

we obtain the desired uniform interior sphere condition with radius $\frac{R}{2}$.

Lemma 6.2. $\hat{M}_{T^{*}} \subset \mathcal{H}\left(x_{0}\right)$ is a convex body in a hemisphere. 
Proof. Let $y_{0} \in \operatorname{int} \hat{M}_{0}$ be arbitrary but fixed. In view of Lemma 5.1 we know that the weakly convex body of $M_{T^{*}}$ with respect to $y_{0}$ can be described as

$$
\hat{M}_{T^{*}}=\varlimsup_{t \in\left[0, T^{*}\right)} \hat{M}_{t} .
$$

In view of the monotonicity, see Lemma 5.1 and Lemma 3.9, we obtain that $\bigcup_{t \in\left[0, T^{*}\right)} \hat{M}_{t}$ is a convex set in a hemisphere. Lemma 6.1 and Theorem 3.5 imply that $\hat{M}_{T^{*}} \subset \mathbb{S}^{n+1}$ is a convex body in a hemisphere.

The lemmata of this section show, that $\hat{M}_{T^{*}}$ satisfies the requirements of Theorem 1.1. Finally we will show that $\hat{M}_{T^{*}}$ is not contained in an open hemisphere:

LEMma 6.3. There is no hemisphere $\mathcal{H}\left(y_{0}\right)$, such that

$$
\hat{M}_{T^{*}} \equiv \overline{\bigcup_{t \in\left[0, T^{*}\right)} \hat{M}_{t}} \subset \operatorname{int} \mathcal{H}\left(y_{0}\right) .
$$

Proof. Suppose contrarily that there existed such a hemisphere. We may assume without loss of generality that $y_{0} \in \operatorname{int} \hat{M}_{T^{*}}$, compare [24]. Thus we may parametrize the surfaces $M_{t}, T<t<T^{*}$, uniformly as graphs over the same equator and may apply Propositions 5.3 and 5.4 to conclude that the flow would exist longer than $T^{*}$.

Hence we obtain:

TheOREM 6.4. There exists $x_{0} \in \mathbb{S}^{n+1}$ such that $M_{T^{*}}=\mathcal{S}\left(x_{0}\right)$.

7. Geometric inequalities. In this section we want to deduce AlexandrovFenchel type inequalities from the convergence of the flow to an equator. We use the inverse mean curvature flow. The mixed volumes of a hypersurface $M$ in the sphere $\mathbb{S}^{n+1}$ are defined for $k \in\{0, \ldots, n\}$ by

$$
V_{k}(M):=\int_{M} \tilde{H}_{k} d \mu
$$

where $\tilde{H}_{k}:=\frac{H_{k}}{\left(\begin{array}{c}n \\ k\end{array}\right)}$ are the normalized elementary symmetric polynomials.

Firstly, we note that a geodesic ball $B_{\rho}$ of radius $0<\rho \leq \frac{\pi}{2}$ satisfies for $k \in$ $\{0, \ldots, n\}$

$$
V_{k}\left(\partial B_{\rho}\right)=\omega_{n} \cos ^{k} \rho \sin ^{n-k} \rho,
$$

where $\omega_{n}$ is the volume of $\mathbb{S}^{n}$. For the sake of brevity, we define $\tilde{V}_{k}:=\frac{V_{k}}{\omega_{n}}$. For geodesic spheres, it is easy to obtain a relation between different mixed volumes. For example there holds

$$
\left(\tilde{V}_{1}\right)^{2}=\left(\tilde{V}_{0}\right)^{2\left(\frac{n-1}{n}\right)}-\left(\tilde{V}_{0}\right)^{2}
$$

and

$$
\tilde{V}_{2}=\left(\tilde{V}_{0}\right)^{\frac{n-2}{n}}-\tilde{V}_{0}
$$

Furthermore, we will establish geometric inequalities between certain quermassintegrals. 
In $\mathbb{S}^{n+1}$ we have the following definition of the quermassintegrals, compare [26] (also for a more detailed definition of the measure $d L_{k}$ ):

Definition 7.1. Let $\hat{M} \subset \mathbb{S}^{n+1}$ be a compact domain. For $k \in\{1, \ldots, n\}$ set

$$
W_{k}(\hat{M})=\frac{(n+1-k) \omega_{k-1} \cdots \omega_{0}}{(n+1) \omega_{n-1} \cdots \omega_{n-k}} \int_{\mathcal{L}_{k}} \chi\left(L_{k} \cap \hat{M}\right) d L_{k},
$$

where $\mathcal{L}_{k}$ is the space of $k$-dimensional totally geodesic subspaces $L$ in $\mathbb{S}^{n+1}, d L_{k}$ is the natural measure on $\mathcal{L}_{k}$ and $\chi$ is the Euler characteristic. Furthermore set

$$
W_{0}(\hat{M})=\operatorname{Vol}(\hat{M})
$$

and

$$
W_{n+1}(\hat{M})=\frac{\omega_{n}}{n+1} \chi(\hat{M})
$$

In Euclidean space the quermassintegrals differ only by constants with respect to the corresponding curvature integrals. This relation is more complicated in curved spaces, however, we still have the following relation between the curvature integrals and the quermassintegrals in the space $\mathbb{S}^{n+1}$, see for example [26, Proposition 7 , Corollary 8] for a proof of this relation.

LEMMA 7.2. If $\hat{M} \subset \mathbb{S}^{n+1}$ is a compact domain with $C^{2}$-boundary, then there holds $W_{1}(\hat{M})=\frac{1}{n+1} V_{0}(\partial M)$ and for $k \in\{1, \ldots, n\}$ there holds

$$
V_{k}(\partial \hat{M})=(n+1)\left(W_{k+1}(\hat{M})-\frac{k}{n+2-k} W_{k-1}(\hat{M})\right) .
$$

Furthermore for $k \in \mathbb{N}$ with $2 k+1 \leq n$ we have

$$
W_{2 k+1}(\hat{M})=\frac{1}{n+1} \sum_{i=0}^{k} \frac{(2 k) ! !(n-2 k) ! !}{(2 k-2 i) ! !(n-2 k+2 i) ! !} V_{2 k-2 i}(\partial \hat{M}) .
$$

We also need the following evolution equations. These evolution equations can be computed by an induction argument using Lemma 4.4 and Lemma 7.2, see also the proof of [23, Thm. B] and [28, Proposition 3.1].

$$
\begin{aligned}
& \frac{d}{d t} \int_{M_{t}} H_{k} d \mu_{t}=\int_{M_{t}}\left((k+1) \frac{H_{k+1}}{H}-(n+1-k) \frac{H_{k-1}}{H}\right) d \mu_{t} . \\
& \frac{d}{d t} W_{k}\left(M_{t}\right)=\frac{n+1-k}{n+1} \int_{M_{t}} \frac{\tilde{H}_{k}}{H} d \mu_{t} .
\end{aligned}
$$

Finally, let us state a decay lemma for the inverse curvature flow.

LEMMA 7.3. For all $1 \leq q<\infty$ there holds

$$
\lim _{t \rightarrow T^{*}} \int_{M_{t}} H^{q}=0
$$

Proof. By the previous results we know

$$
u \rightarrow c
$$


in the $C^{1}$ norm, where we use a graph representation as in Proposition 5.2. The second fundamental form is

$$
h_{i j} v^{-1}=-u_{i j}+\bar{h}_{i j}
$$

where covariant differentiation is performed with respect to the induced metric. Thus

$$
\int_{M_{t}} H v^{-1}=\int_{M_{t}} \bar{h}_{i j} g^{i j}
$$

and

$$
\int_{M_{t}} H=\int_{M_{t}} H\left(1-v^{-1}\right)+\int_{M_{t}}\left(\bar{H}-\frac{\bar{H}}{n}\|D u\|^{2}\right) .
$$

Since

$$
g_{i j}=u_{i} u_{j}+\sin ^{2} u \sigma_{i j},
$$

the volume element is uniformly bounded and thus the right hand side converges to 0 . The other $L^{q}$ norms converge to 0 by interpolation.

This leads to our first geometric inequality:

TheOREM 7.4. Let $M \subset \mathbb{S}^{n+1}$ be an embedded, closed, connected and convex $C^{2}$-hypersurface. Then we have the inequality

$$
\left(\tilde{V}_{1}(M)\right)^{2} \geq\left(\tilde{V}_{0}(M)\right)^{2\left(\frac{n-1}{n}\right)}-\left(\tilde{V}_{0}(M)\right)^{2},
$$

and equality holds if and only if $M$ is a geodesic sphere.

Proof. First of all, we can assume that the hypersurface is smooth and strictly convex, since otherwise we can use convolutions as in the proof of Corollary 1.2 to obtain a sequence of approximating smooth, strictly convex hypersurfaces converging in $C^{2}$ to $M$.

We consider the flow of the initial hypersurface $M$ by the inverse mean curvature. Let $M_{t}, t \in\left[0, T^{*}\right)$, be the level hypersurfaces of the flow, where we know that $M_{T^{*}}$ is a geodesic sphere with radius $\frac{\pi}{2}$ and the graphs around the center of this sphere, $M_{t}=$ graph $\left.\right|_{\mathbb{S}^{n}} u(t, \cdot)$, converge in $C^{1}$ to $u\left(T^{*}, \cdot\right) \equiv \frac{\pi}{2}$. Furthermore we know that the mean curvature of the level hypersurfaces converges almost everywhere to 0 by Lemma 7.3.

For $t \in\left[0, T^{*}\right]$ we define

$$
\phi(t):=\frac{\left(V_{1}\left(M_{t}\right)\right)^{2}}{\left(V_{0}\left(M_{t}\right)\right)^{2\left(\frac{n-1}{n}\right)}}+\left(V_{0}\left(M_{t}\right)\right)^{\frac{2}{n}} .
$$

Then $\phi(t) \rightarrow\left(V_{0}\left(B_{\frac{\pi}{2}}\right)\right)^{\frac{2}{n}}$ for $t \rightarrow T^{*}$, since $V_{0}\left(M_{t}\right) \rightarrow V_{0}\left(M_{T^{*}}\right)$ for $t \rightarrow T^{*}$ in view of the $C^{1}$-convergence and $V_{1}\left(M_{t}\right) \rightarrow 0$ for $t \rightarrow T^{*}$ in view of the convergence of the mean curvature to zero almost everywhere.

Hence if we can show that $\phi$ is monotonically non-increasing, we obtain

$$
\frac{\left(V_{1}(M)\right)^{2}}{\left(V_{0}(M)\right)^{2\left(\frac{n-1}{n}\right)}}+\left(V_{0}(M)\right)^{\frac{2}{n}}=\phi(0) \geq \phi\left(T^{*}\right)=\omega_{n}^{\frac{2}{n}} .
$$

This implies the geometric inequality. 
We have in view of (7.10) and (iii) from Lemma 4.1:

$$
\begin{aligned}
& \left(V_{0}\left(M_{t}\right)\right)^{2\left(\frac{n-1}{n}\right)+1} \frac{d \phi}{d t} \\
= & 2 V_{0}\left(M_{t}\right) \int_{M_{t}} \frac{H}{n} d \mu_{t} \int_{M_{t}}\left(\frac{2 H_{2}}{n H}-\frac{1}{H}\right) d \mu_{t} \\
& -2 \frac{n-1}{n^{3}}\left(\int_{M_{t}} H d \mu_{t}\right)^{2} \int_{M_{t}} 1 d \mu_{t}+\frac{2}{n}\left(V_{0}\left(M_{t}\right)\right)^{2} \int_{M_{t}} 1 d \mu_{t} \\
\leq & \frac{2}{n} V_{0}\left(M_{t}\right)\left(\left(V_{0}\left(M_{t}\right)\right)^{2}-\int_{M_{t}} H d \mu_{t} \int_{M_{t}} \frac{1}{H} d \mu_{t}\right) \leq 0 .
\end{aligned}
$$

In the last inequality we have used the Cauchy-Schwarz inequality and hence the inequality is strict unless $H$ is constant on $M_{t}$. Hence we obtain that $\phi$ is monotonically decreasing unless $M$ is a geodesic sphere.

We can also prove a geometric inequality relating $\tilde{V}_{2}$ and $\tilde{V}_{0}$.

TheOREM 7.5. Let $n \geq 3$ and $M \subset \mathbb{S}^{n+1}$ be an embedded, closed, connected and convex $C^{2}$-hypersurface. Then we have the inequality

$$
\tilde{V}_{2}(M) \geq\left(\tilde{V}_{0}(M)\right)^{\frac{n-2}{n}}-\tilde{V}_{0}(M)
$$

and equality holds if and only if $M$ is a geodesic sphere.

Proof. Again we assume $M$ to be smooth and strictly convex and we use the same curvature flow as in the proof of Theorem 7.4. For $t \in\left[0, T^{*}\right]$ we define

$$
\phi(t):=\frac{V_{2}\left(M_{t}\right)+V_{0}\left(M_{t}\right)}{\left(V_{0}\left(M_{t}\right)\right)^{\frac{n-2}{n}}} .
$$

Again we have to show that $\phi$ is monotonically non-increasing to obtain the geometric inequality (7.22). We have in view of (7.10):

$$
\begin{aligned}
\left(V_{0}\left(M_{t}\right)\right)^{\frac{n-2}{n}+1} \frac{d \phi}{d t}= & V_{0}\left(M_{t}\right) \int_{M_{t}} \frac{3 H_{3}}{\left(\begin{array}{c}
n \\
2
\end{array}\right) H}+V_{0}\left(M_{t}\right) \int_{M_{t}}\left(1-\frac{(n-1)}{\left(\begin{array}{c}
n \\
2
\end{array}\right)}\right) d \mu_{t} \\
& -\frac{n-2}{n} \int_{M_{t}} 1 d \mu_{t}\left(\int_{M_{t}} \tilde{H}_{2} d \mu_{t}+V_{0}\left(M_{t}\right)\right) \\
= & \frac{V_{0}\left(M_{t}\right)}{\left(\begin{array}{c}
n \\
2
\end{array}\right)} \int_{M_{t}}\left(\frac{3 H_{3}}{H}-\frac{n-2}{n} H_{2}\right) d \mu_{t} \leq 0,
\end{aligned}
$$

where the last inequality follows from (iii) from Lemma 4.1. Again, this inequality is strict unless the hypersurface is totally umbilic, which implies that $\phi$ is only stationary for geodesic spheres.

TheOrem 7.6. Let $M \subset \mathbb{S}^{n+1}$ be an embedded, closed, connected and convex $C^{2}$-hypersurface. Let $k \in \mathbb{N}_{+}$with $2 k+1 \leq n$ and let $\hat{M}$ be the convex body enclosed by $M$. Then we have the inequality

$$
W_{2 k+1}(\hat{M}) \geq \frac{\omega_{n}}{n+1} \sum_{i=0}^{k}(-1)^{i} \frac{n-2 k}{n-2 k+2 i}\left(\begin{array}{l}
k \\
i
\end{array}\right)\left(\frac{n+1}{\omega_{n}} W_{1}(\hat{M})\right)^{\frac{n-2 k+2 i}{n}}
$$

and equality holds if and only if $M$ is a geodesic sphere. 
Proof. For the sake of brevity, let us denote the right hand side of the equation (7.25) by $A_{k}(\hat{M})$.

We prove the result by induction on $k$. For $k=1$ the result follows from Theorem 7.5 and Lemma 7.2. Let us assume we have proved the result for $k-1 \in \mathbb{N}$ with $2 k+1 \leq n$.

We assume the hypersurface to be smooth and strictly convex and use the same curvature flow as in the proof of Theorem 7.4. For $t \in\left[0, T^{*}\right]$ we define

$$
\phi(t):=\frac{W_{2 k+1}\left(\hat{M}_{t}\right)-A_{k}\left(\hat{M}_{t}\right)}{W_{1}\left(\hat{M}_{t}\right)^{\frac{n-2 k}{n}}} .
$$

From now on we will drop the arguments and write simply $W_{k}$ instead of $W_{k}(\hat{M})$. We will show that $\phi$ is monotonically non-increasing. We obtain from (7.11) and Lemma 7.2 :

$$
\begin{aligned}
\frac{d}{d t} \phi & \leq \frac{(n-2 k)}{n(n+1)} \frac{\int_{M_{t}} \tilde{H}_{2 k} d \mu_{t}-(n+1) W_{2 k+1}}{W_{1}^{\frac{n-2 k}{n}}}-\frac{d}{d t}\left(\frac{A_{k}}{W_{1}^{\frac{n-2 k}{n}}}\right) \\
& =-\frac{(n-2 k) 2 k}{n(n+2-2 k)} \frac{W_{2 k-1}}{W_{1}^{\frac{n-2 k}{n}}}-\frac{d}{d t}\left(\frac{A_{k}}{W_{1}^{\frac{n-2 k}{n}}}\right) .
\end{aligned}
$$

Note that the inequality at time $t$ is an equality if and only $M_{t}$ is totally umbilic. Using the induction hypothesis we obtain after a simple calculation

$$
\frac{d}{d t} \phi \leq 0
$$

again with equality if and only if $M_{t}$ is totally umbilic. Employing (7.9) and the decay Lemma, we obtain

$$
\begin{aligned}
W_{2 k+1}\left(\hat{M}_{t}\right)-A_{k}\left(\hat{M}_{t}\right) & \rightarrow \frac{\omega_{n}}{n+1} \frac{(2 k) ! !(n-2 k) ! !}{n ! !} \\
& -\frac{\omega_{n}}{n+1} \sum_{i=0}^{k}(-1)^{i} \frac{n-2 k}{n-2 k+2 i}\left(\begin{array}{c}
k \\
i
\end{array}\right)
\end{aligned}
$$

for $t \rightarrow T^{*}$. However, the right hand side equals zero, as a proof by induction shows. Hence we obtain the inequality (7.25) and equality holds if and only if $M$ is a geodesic sphere.

Acknowledgements. We would like to thank Prof. Oliver Schnürer for an interesting discussion concerning the interior sphere condition. We would like to thank Prof. Claus Gerhardt for pointing out, that the class of curvature functions for which Theorem 1.4 is true had to be modified. Furthermore we would like to thank Prof. Rolf Schneider for valuable comments on isoperimetric problems for the mixed volumes.

\section{REFERENCES}

[1] N. Ando, Strict convexity of hypersurfaces in spheres, J. Math. Sci., 5:3 (1998), pp. 423-433.

[2] B. Andrews, Pinching estimates and motion of hypersurfaces by curvature functions, J. Reine Angew. Math., 608 (2007), pp. 17-33.

[3] G. Bredon, Topology and Geometry, Graduate Texts in Mathematics, Springer, 1993. 
[4] L. L. De Lima and F. Girao, An Alexandrov-Fenchel-type inequality in hyperbolic space with an application to a Penrose inequality, Ann. Henri Poincaré, 17:4 (2016), pp. 979-1002.

[5] M. Do Carmo And F. Warner, Rigidity and convexity of hypersurfaces in spheres, J. Differ. Geom., 4:2 (1970), pp. 133-144.

[6] O. Ferreira, A. Iusem, And S. Nemeth, Projections onto convex sets on the sphere, J. Glob. Optim., 57:3 (2013), pp. 663-676.

[7] Y. Ge, G. Wang, and J. Wu, Hyperbolic Aleksandrov-Fenchel quermassintegral inequalities 1, unpublished, 032013.

[8] _ Hyperbolic Aleksandrov-Fenchel quermassintegral inequalities 2, J. Differ. Geom., 98:2 (2014), pp. 237-260.

[9] C. Gerhardt, Flow of nonconvex hypersurfaces into spheres, J. Differ. Geom., 32:1 (1990), pp. 299-314.

[10] $ـ$ Curvature problems, Series in Geometry and Topology, vol. 39, International Press of Boston Inc., 2006.

[11] _ Curvature flows in semi-Riemannian manifolds, Geometric flows (Huai-Dong Cao and Shing-Tung Yau, eds.), Surveys in Differential Geometry, vol. 12, International Press of Boston Inc., 2007, pp. 113-166.

[12] - Inverse curvature flows in hyperbolic space, J. Differ. Geom., 89:3 (2011), pp. 487-527.

[13] - Non-scale-invariant inverse curvature flows in Euclidean space, Calc. Var. Partial Differ. Equ., 49:1-2 (2014), pp. 471-489.

[14] Curvature flows in the sphere, J. Differ. Geom., 100:2 (2015), pp. 301-347.

[15] P. GuAn And J. LI, The quermassintegral inequalities for $k$-convex starshaped domains, Adv. Math., 221:5 (2009), pp. 1725-1732.

[16] G. Huisken, Flow by mean curvature of convex surfaces into spheres, J. Differ. Geom., 20:1 (1984), pp. 237-266.

[17] G. Huisken and T. Ilmanen, The inverse mean curvature flow and the Riemannian Penrose inequality, J. Differ. Geom., 59:3 (2001), pp. 353-437.

[18] G. Huisken and C. Sinestrari, Convexity estimates for mean curvature flow and singularities of mean convex surfaces, Acta Math., 183:1 (1999), pp. 45-70.

[19] N. KRYLOV, Nonlinear elliptic and parabolic equations of the second order, Mathematics and its applications, vol. 7, Springer, 1987.

[20] G. Lieberman, Second order parabolic differential equations, World Scientific, 1998.

[21] M. Makowski, Mixed volume preserving curvature flows in hyperbolic space, unpublished, 08 2012.

[22] J. A. McCoy, Mixed volume preserving curvature flows, Calc. Var. Partial Differ. Equ., 24:2 (2005), pp. 131-154.

[23] R. REILly, Variational properties of functions of the mean curvatures for hypersurfaces in space forms, J. Differ. Geom., 8:3 (1973), pp. 465-477.

[24] L. Santalo, Convex regions on the n-dimensional spherical surface, Ann. Math., 47:3 (1946), pp. $448-459$.

[25] J. Scheuer, Non-scale-invariant inverse curvature flows in hyperbolic space, Calc. Var. Partial Differ. Equ., 53:1-2 (2015), pp. 91-123.

[26] G. SolAnes, Integral geometry and the Gauss-Bonnet theorem in constant curvature spaces, Trans. Am. Math. Soc., 358:3 (2006), pp. 1105-1115.

[27] J. URBAS, On the expansion of starshaped hypersurfaces by symmetric functions of their principal curvatures, Math. Z., 205:1 (1990), pp. 355-372.

[28] G. WANG AND C. XIA, Isoperimetric type problems and Alexandrov-fenchel type inequalities in the hyperbolic space, Adv. Math., 259 (2014), pp. 532-556.

[29] Y. Wei And C. XIong, Alexandrov-Fenchel type inequalities for convex hypersurfaces in hyperbolic space and in sphere, Pac. J. Math., 277:1 (2015), pp. 219-239. 